\title{
Self-T-Dual Brane Cosmology and the Cosmological Constant Problem
}

\author{
Olindo Corradini \\ Dipartimento di Fisica, Università di Bologna \\ and INFN, Sezione di Bologna \\ Via Irnerio, 46 - Bologna I-40126, Italy \\ E-mail: olindo.corradini@bo.infn.it \\ Massimiliano Rinaldi \\ School of Mathematical Sciences, \\ University College Dublin, \\ Belfield, Dublin 4, Ireland \\ E-mail: massimiliano.rinaldi@ucd.ie
}

\begin{abstract}
We consider a codimension-one brane embedded in a gravity-dilaton bulk action, whose symmetries are compatible with T-duality along the space-like directions parallel to the brane, and the bulk time-like direction. The equations of motions in the string frame allow for a smooth background obtained by the union of two symmetric patches of AdS space. The Poincaré invariance of the solution appears to hold independently of the value of the brane vacuum energy, through a self-tuning property of the dilaton ground state. Moreover, the effective cosmology displays a bounce, at which the scale factor does not shrink to zero. Finally, by exploiting the T-duality symmetry, we show how to construct an ever-expanding Universe, along the lines of the Pre-Big Bang scenario.
\end{abstract}

KEywords: String Theory and Cosmology. 


\section{Contents}

1. Introduction 1

2. Background geometry and time-like T-duality 3

3. Invariant junction conditions 7

4. Brane Cosmology 8

4.1 Pure tension: $\omega=-1$

4.2 Perfect fluid: $\omega \neq-1$

4.3 Pre-Big Bang Scenario 12

5. Discussion 13

A. Extrinsic curvature and its dual 14

\section{Introduction}

The major problem associated with the Randall-Sundrum (RS) models [1], 2] is probably represented by the fine-tuning between the vacuum expectation value of the brane fields and the bulk cosmological constant, which is required to ensure that the background is the union of smooth AdS patches.

An attempt to tackle the fine-tuning problem was realised by Kachru, Schulz, and Silverstein (KSS) by introducing a bulk scalar field [3] (see also [4]). The addition of this extra degree of freedom lifts the fine-tuning to a less restrictive self-tuning mechanism, in which the dilaton field adjusts spontaneously in such a way that Poincaré 4-dimensional solutions exist, no matter what the value of the brane vacuum energy is. 1

The KSS model suffers from a serious drawback, namely the existence of naked singularities in the bulk. These, however, can be treated in a few ways. For example, Low and Zee [6] have shown that upon adding a bulk Gauss-Bonnet combination and a bulk (super-)potential one can make the singularity disappear, but this leads again to fine-tuned parameters. ${ }^{2}$ Alternatively, the naked singularity can become harmless

\footnotetext{
${ }^{1}$ See [5] for a (incomplete) list of interesting alternative attempts to solve the fine-tuning problem within the brane-world scenario.

${ }^{2} \mathrm{~A}$ detailed discussion upon self-tuning models can be found in [7].
} 
if one considers time-dependent bulks, as first noted in [8] (see also [9]). Indeed, in this case the singularity becomes null and cannot be reached by the brane. However, the bulk space-time must be cut off by hand at the singularity, in order to have a finite effective Planck constant. The possibility of screening the bulk singularity behind a horizon was shown to lead to a no-go theorem, unless some space-curvature is given to the brane [10]. Also, the presence of a curvature singularity compromises the truncation of the effective action to its lowest order, and needs the inclusion of bulk higher-curvature terms.

In this Letter, we reconsider some of these issues from the point of view of the string frame. The motivation relies on the assumption that the brane lives in a background obtained by some compactification of string theory. Therefore, there might be inherited string dualities, which can play an important rôle in the brane dynamics. If these dualities are taken into account, they should then be manifest in the string frame. In particular, we consider T-duality as the underlying symmetry that must be preserved not only by the equation of motions for the bulk, but also by the junction conditions associated to the brane. This approach was already adopted in [11], and further developed in [12], where a strong connection with the Pre-Big Bang (PBB) scenario was discovered. Here, we extend the results of 12 to the case when T-duality acts also along the bulk time direction. As explained in more detail below, the time-like T-duality is motivated by the choice of a warped metric as background geometry, which is Poincaré invariant along the brane coordinates.

It is known that T-duality is a mapping between type IIA and type IIB string theory. At low energy, this symmetry still holds at the level of the equations of motion [13]. In particular, when all the forms of the RR sector of the theory are switched off, the action becomes self-T-dual. With this we mean that T-duality becomes a mapping between different solutions to the equations of motion derived from the same action, a fact which lies at the foundations of the PBB scenario [14, 15].

T-duality along the time direction is known to map type II (A or B) to type II* (B or A) string theory [16]. Also in this case, when all forms are switched off, type

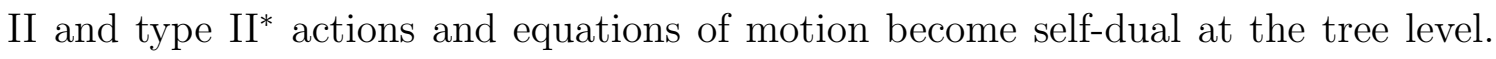
In general, time-like T-duality requires the time direction to be compact, and this can be obtained by means of analytic continuation (see for example [17). In our case, we show instead that the duality symmetry is meaningful because one of the possible bulk geometry is actually AdS, which is known to contain closed time-like curves. This solution is particularly important because, as we will see, it is Poincaré invariant along the brane directions, and it does not require any fine-tuning between bulk and brane parameters.

The insertion of a codimension-one brane in the bulk geometry requires junction conditions, which must be satisfied by the metric components and by the dilaton, upon specification of the energy-momentum tensor of the localised matter. These 
conditions lead to the effective cosmological equations, which govern the evolution of the Universe as seen by an observer living on the brane. In this work, we assume that also these equations must be invariant under T-duality, and this leads to certain transformation laws for the brane matter fields. These are different from the ones found in [12] (where only space-like duality was considered), which are similar to the usual transformation laws of the PBB scenario.

Throughout this Letter, we refer to the brane embedded in a self-T-dual bulk as "self-T-dual brane". This terminology is justified by the fact that if we take an NS5-brane as a prototype for our brane, then T-duality along directions longitudinal to the world-volume of the brane leaves it unchanged. Indeed, the T-dual partner is again an NS5-brane wrapped on the dual directions (see e.g. [18] for a general discussion upon solitonic objects in string theory).

At the level of the effective brane cosmology, we find both static and evolving models of the Universe, together with their dual descriptions. In particular, the dynamical models display the same bouncing behaviour as their dual counterpart. An alternative to the bouncing cosmology can be built by "gluing" part of the solution to its dual, along the lines of the PBB scenario. In this way, one obtains an ever expanding cosmological model. This construction seems also to suggest the possibility of a phase-transition between a (pre-bounce) solution with non-localised brane gravity, and a (post-bounce) solution with localised gravity. However, there are some caveats about this construction, which will be pointed out.

The structure of this work is the following: in Sec. 2 we consider the bulk geometry in string frame, and we show that we can find a self-T-dual AdS background. In Sec. 3 we study the junction conditions induced by the insertion of a brane in the bulk. By imposing the self-T-duality on the junction equations, we find the transformation laws of the energy density and of the pressure, in the case when the brane matter is taken to be a perfect fluid. Equipped with these information, we then turn our attention to the brane cosmological equations in Sec. 目, where we find the various cosmological solutions mentioned above. We finally end this Letter with some remarks and open problems.

\section{Background geometry and time-like T-duality}

We consider the D-dimensional action in string the frame given by

$$
\begin{aligned}
S=S_{\text {bulk }}+S_{\text {brane }}= & \frac{1}{2} \int_{\mathcal{M}} d^{D} x \sqrt{-g} e^{-2 \phi}\left[R+4(\nabla \phi)^{2}-V\right]+ \\
& -\int_{\Sigma} d^{D-1} x \sqrt{-h} e^{-2 \phi}\left[K^{ \pm}-\mathcal{L}\right],
\end{aligned}
$$

where $K^{ \pm}=K^{+}+K^{-}$is the extrinsic curvature on the two sides of the brane submanifold $\Sigma$ embedded in the bulk spacetime $\mathcal{M}$. Also, $h_{\mu \nu}=g_{\mu \nu}-n_{\mu} n_{\nu}$ is the 
induced metric on $\Sigma$, where $n^{A}$ are the components of the normal vector pointing into the bulk. We assume that the bulk metric has the form

$$
d s^{2}=g_{M N} d x^{M} d x^{N}=e^{2 \sigma(z)} \eta_{\mu \nu} d x^{\mu} d x^{\nu}+d z^{2}
$$

where $\eta_{\mu \nu} d x^{\mu} d x^{\nu}$ stands for the Minkowski space-time of dimension $D-1$. This metric is clearly invariant under translations along $x^{\mu}$. Therefore, by assuming that the T-duality transformation is allowed along the time coordinate, we expect the equations of motion (and, eventually, the action) to be invariant under

$$
\sigma(z) \stackrel{\mathrm{T}}{\longrightarrow}-\sigma(z), \quad \phi \stackrel{\mathrm{T}}{\longrightarrow} \phi-(D-1) \sigma,
$$

according to Buscher's rules [13]. To prove this, we consider first the Ricci scalar, which reads

$$
g^{M N} R_{M N}=-(D-1)\left(2 \sigma^{\prime \prime}+D \sigma^{2}\right),
$$

where the prime denotes differentiation with respect to $z$. By defining the shifted dilaton as

$$
\bar{\phi}=\phi-\frac{D-1}{2} \sigma
$$

and after partial integration, we can write the bulk part of the action (2.1) as

$$
\begin{aligned}
S_{\mathrm{bulk}}= & \frac{1}{2} \int_{\mathcal{M}} d^{D} x e^{-2 \bar{\phi}}\left[4 \bar{\phi}^{\prime 2}-(D-1) \sigma^{2}-V\right]+ \\
& -(D-1) \int_{\partial \mathcal{M}} d^{D-2} x d t \sigma^{\prime} e^{-2 \bar{\phi}} .
\end{aligned}
$$

If the induced metric has the form

$$
h_{\mu \nu} d x^{\mu} d x^{\nu}=-d \tau^{2}+e^{2 \sigma(z)} \delta_{i j} d x^{i} d x^{j}
$$

it follows that $\sqrt{-h} e^{-2 \phi}=e^{-\sigma} e^{-2 \bar{\phi}}$, and the boundary part of the action reads

$$
S_{\text {boundary }}=-\int_{\Sigma} d^{D-2} x d \tau e^{-\sigma} e^{-2 \bar{\phi}} 2 K
$$

where we also assumed a $Z_{2}$ symmetry along the transverse direction. With the help of the normalisation condition

$$
d t=e^{-\sigma \sqrt{1+\dot{z}^{2}}} d \tau
$$

we find that the full action reads

$$
\begin{aligned}
S= & \frac{1}{2} \int_{\mathcal{M}} d^{D} x e^{-2 \bar{\phi}}\left[4 \bar{\phi}^{\prime 2}-(D-1) \sigma^{2}-V\right]+ \\
& -\int_{\partial \mathcal{M}} d^{D-2} x d t e^{-2 \bar{\phi}}\left[2(D-1) \sigma^{\prime}+\frac{2 K-\mathcal{L}}{\sqrt{1+\dot{z}^{2}}}\right] .
\end{aligned}
$$


Given that, by definition, the shifted dilaton is unchanged by T-duality, it is evident that the bulk part of the action is invariant when $V$ is a function of $\bar{\phi}$ only. The boundary part is also invariant, as this follows from the transformation law of the trace of the extrinsic curvature (see the Appendix)

$$
K \stackrel{\mathrm{T}}{\rightarrow} \tilde{K}=K+2(D-1) \sigma^{\prime} \sqrt{1+\dot{z}^{2}}
$$

and from the fact that $\sqrt{1+\dot{z}^{2}}=-n_{z}$ does not change under T-duality. However, what really matters here is whether the bulk equations of motion are invariant under T-duality. This is indeed the case, as it can clearly be seen from the expressions

$$
\begin{aligned}
& 0=\bar{\phi}^{\prime \prime}-\bar{\phi}^{\prime 2}-\frac{D-1}{4} \sigma^{2}+\frac{1}{8}\left(\frac{\partial V}{\partial \bar{\phi}}-2 V\right), \\
& 0=\frac{d}{d z}\left(\sigma^{\prime} e^{-2 \bar{\phi}}\right),
\end{aligned}
$$

obtained by variation of the action with respect to $\sigma$ and $\bar{\phi}$.

These equations can be easily solved if we assume that $V$ is an exponential function of $\bar{\phi}$. Potentials of this type were first introduced in the context of string cosmology in order to obtain a regular curvature bounce [19]-[20]. In general, such potentials are not invariant under general coordinate transformations. However, a covariant formulation for the shifted dilaton (and hence for the potential) was recently proposed in 21]. In these works, it is also argued that exponential potentials of the shifted dilaton can be generated by string loop corrections, as $e^{2 \bar{\phi}}$ is interpreted as the "reduced" - i.e. one-dimensional - string coupling constant. The generally covariant shifted dilaton is then defined as

$$
e^{-2 \bar{\phi}}=2 \int d^{D} y \sqrt{-g(y)} e^{-2 \phi(y)} \sqrt{\partial_{\mu} \phi(y) \partial^{\mu} \phi(y)} \delta(\phi(x)-\phi(y)),
$$

and the equations of motion are modified accordingly. In our case, it turns that the above definition for the shifted dilaton coincides with Eq. (2.6), and the equations of motion are given by

$$
\begin{aligned}
0 & =\bar{\phi}^{\prime \prime}-\bar{\phi}^{\prime 2}-\frac{D-1}{4} \sigma^{2}+V_{0} e^{\beta \bar{\phi}}, \\
\sigma^{\prime} & =k e^{2 \bar{\phi}}
\end{aligned}
$$

where we assumed that

$$
V=\frac{8 V_{0}}{\beta-2} e^{\beta \bar{\phi}}
$$

In these expressions, $V_{0}, \beta$, and $k$ are constants. We consider the ansatz solution $\bar{\phi}=\alpha \ln \left(z-z_{0}\right)+\bar{\phi}_{0}$. By substituting this expression, together with the second equation of motion, into the first, we find

$$
4 \alpha(\alpha+1)+k^{2}(D-1)\left(z-z_{0}\right)^{4 \alpha+2} e^{4 \bar{\phi}_{0}}-4 V_{0}\left(z-z_{0}\right)^{\alpha \beta+2} e^{\beta \bar{\phi}_{0}}=0 .
$$


The analysis of this equation is straightforward and leads to three type of solutions, namely

$$
\begin{array}{lll}
\alpha=-\frac{1}{2}, & \beta=4, & k^{2}=\frac{1}{D-1}\left(4 V_{0}+e^{-4 \bar{\phi}_{0}}\right), \\
\alpha=-1, & \beta=4, & k^{2}=\frac{4}{D-1} V_{0}, \\
\alpha=0, & \forall \beta, & k^{2}=\frac{4}{D-1} V_{0} e^{(\beta-4) \bar{\phi}_{0}},
\end{array}
$$

where in the last two cases $V_{0}>0$. By studying the Ricci scalar and higher order curvature invariants, it turns out that the first two solutions carry a naked singularity at $z=0$.

The last solution however is singularity-free. Indeed, the background is AdS as the Ricci scalar reads

$$
\mathcal{R}=-D(D-1) \lambda^{2}<0
$$

where

$$
\lambda=2\left(\frac{V_{0}}{D-1}\right)^{\frac{1}{2}} e^{\frac{\beta \bar{\phi}_{0}}{2}} .
$$

Also, the shifted dilaton becomes constant, while the warp factor and the dilaton read respectively

$$
\begin{aligned}
& \sigma(z)= \pm \lambda\left(z-z_{0}\right) \\
& \phi(z)= \pm \frac{D-1}{2} \lambda\left(z-z_{0}\right)+\bar{\phi}_{0},
\end{aligned}
$$

for arbitrary $z_{0}$. It is interesting to note that the bulk potential is now constant, but the AdS solution is insensitive to its sign (which is determined by the arbitrary quantity $\beta-2$ ). However, the value of $\beta$ can be fixed if one allows for variations of the metric element $g_{z z}$. Then, there is a further equation of motion, known as the "zero energy condition" (see for example [19]), which reads

$$
4 \bar{\phi}^{\prime 2}-(D-1) \sigma^{2}+V=0 \text {. }
$$

In our case, the net effect of this constraint is that $\beta=4$, which implies a positive potential. Notice that $\beta=4$ corresponds to a two-loop potential in the language of [21].

In summary, the metric and the dilaton field, corresponding to the solution $\sigma=\lambda\left(z-z_{0}\right)$, are given respectively by

$$
d s^{2}=e^{2 \lambda\left(z-z_{0}\right)} \eta_{\mu \nu} d x^{\mu} d x^{\nu}+d z^{2}, \quad \phi(z)=\frac{D-1}{2} \lambda\left(z-z_{0}\right)+\bar{\phi}_{0},
$$


while their dual counterparts, corresponding to the solution $\tilde{\sigma}=-\lambda\left(z-z_{0}\right)$, are

$$
d \tilde{s}^{2}=e^{-2 \lambda\left(z-z_{0}\right)} \eta_{\mu \nu} d x^{\mu} d x^{\nu}+d z^{2}, \quad \tilde{\phi}(z)=-\frac{D-1}{2} \lambda\left(z-z_{0}\right)+\bar{\phi}_{0} .
$$

From these expressions, it becomes clear that the dual counterparts represent the same physical solution, because the T-duality transformation is in fact equivalent to the mere change of coordinate $z \rightarrow-z$. However, as we will see below, this equivalence does not hold when a brane with $Z_{2}$ symmetry is inserted in the background.

\section{Invariant junction conditions}

We now turn our attention to the case when a brane is embedded in the background. As mentioned in the introduction, the idea is to impose the invariance under $\mathrm{T}$ duality of the equations of motion and of the junction conditions. These can be written in string frame as (see [4, 11, 22])

$$
\begin{aligned}
K_{\mu \nu} & =-\frac{1}{2} T_{\mu \nu}-\frac{1}{4} h_{\mu \nu} T^{\phi}, \\
n^{A} \partial_{A} \phi & =\frac{K}{2}+\frac{T^{\phi}}{8}
\end{aligned}
$$

where

$$
\begin{aligned}
T^{\phi} & =e^{2 \phi} \frac{\delta\left(e^{-2 \phi} \mathcal{L}\right)}{\delta \phi}, \\
T_{\mu \nu} & =-\frac{2}{\sqrt{-h}} \frac{\delta \sqrt{-h} \mathcal{L}}{\delta h^{\mu \nu}} .
\end{aligned}
$$

The invariance under T-duality imposes constraints on the transformation law of $T^{\phi}$ and $T_{\mu \nu}$. To see this, we write Eq. (3.2) in terms of the shifted dilaton (2.6), and we use the explicit expression for the trace of $K$ (see Appendix A). Thus, we find

$$
n^{z} \bar{\phi}^{\prime}-\frac{1}{2} \frac{d n^{z}}{d z}=\frac{T^{\phi}}{8} .
$$

Since $\bar{\phi}$ and $n^{z}$ are invariant under T-duality, we have that

$$
T^{\phi} \stackrel{\mathrm{T}}{\longrightarrow} \tilde{T}^{\phi}=T^{\phi} .
$$

If we assume that the matter on the brane behaves as a perfect fluid, namely $T_{\nu}^{\mu}=$ $\operatorname{diag}(-\mu, p, p, \ldots, p)$ and $\tilde{T}_{\nu}^{\mu}=\operatorname{diag}(-\tilde{\mu}, \tilde{p}, \tilde{p}, \ldots, \tilde{p})$, it follows, from the $i j$ and $\tau \tau$ components of Eq. (3.1), that

$$
\begin{aligned}
& \mu \stackrel{\mathrm{T}}{\longrightarrow} \tilde{\mu}=\mu+2 p+T^{\phi}, \\
& p \stackrel{\mathrm{T}}{\longrightarrow} \tilde{p}=-p-T^{\phi} .
\end{aligned}
$$


Note that the last two conditions also imply that

$$
\tilde{\mu}+\tilde{p}=\mu+p .
$$

Therefore, if $p=\omega \mu$ and $\tilde{p}=\tilde{\omega} \tilde{\mu}$, and $\omega=-1$, then $\tilde{\omega}=-1$ as well, independently of the value of $T^{\phi}$.

The junction conditions can be written in the form of cosmological equations by using the explicit components of the extrinsic curvature, and by defining the Hubble parameter as $H=\dot{z} \sigma^{\prime}=\partial_{\tau} \ln A$, where $A(\tau)$ is the scale factor. Indeed, from the $i j$ component of Eq. (3.1), we find the effective Friedmann equation

$$
H^{2}=\frac{1}{4}\left(p+\frac{1}{2} T^{\phi}\right)^{2}-\left(\frac{A^{\prime}}{A}\right)^{2},
$$

while the $\tau \tau$ component of Eq. (3.1) yields the evolution equation of $\mu$ and $p$

$$
(\mu+p) H-\left(p+\frac{1}{2} T^{\phi}\right) \frac{d}{d \tau} \ln \frac{A^{\prime}}{A}+\frac{d}{d \tau}\left(p+\frac{1}{2} T^{\phi}\right)=0 .
$$

Finally, the junction condition on the dilaton leads to

$$
(H+2 \dot{\bar{\phi}})\left(p+\frac{1}{2} T^{\phi}\right)+\mu H=0 .
$$

\section{Brane Cosmology}

The cosmological equations seen in the previous section simplify considerably when the bulk geometry is determined by the AdS solution found in Sec. 2. Indeed, if we set $\bar{\phi}=\bar{\phi}_{0}$ and $A=e^{ \pm \lambda\left(z-z_{0}\right)}$, Eqs. (3.10)-(3.12) reduce respectively to

$$
\begin{aligned}
H^{2} & =\frac{1}{4} \mu^{2}-\lambda^{2}, \\
\dot{\mu} & =(\mu+p) H, \\
0 & =\mu+p+\frac{1}{2} T^{\phi} .
\end{aligned}
$$

The last equation is always compatible with Eq. (3.6) and with Eq. (3.9), hence the set of transformation rules under T-duality reduce to

$$
\mu \stackrel{\mathrm{T}}{\longrightarrow} \tilde{\mu}=-\mu \quad p \stackrel{\mathrm{T}}{\longrightarrow} \tilde{p}=p+2 \mu, \quad \Leftrightarrow \quad \omega \stackrel{\mathrm{T}}{\longrightarrow} \tilde{\omega}=-(2+\omega) .
$$

The first of these transformations signals the violation of the strong energy condition in the duality transition. As we will see below, this problem can be mitigated by 
assuming that the total energy density contains a time-dependent tension which can be positive or negative, along the lines of [8].

From Eq. (4.2), we see that the energy is not conserved on the brane ${ }^{3}$. However, it is possible to find a new frame where the energy is conserved. To see this, we define the conformal induced metric

$$
\gamma_{\mu \nu} d x^{\mu} d x^{\nu}=-d \xi^{2}+E^{2} \delta_{i j} d x^{i} d x^{j}
$$

where $\gamma_{\mu \nu}=e^{-4 \phi /(D-2)} h_{\mu \nu}$, and we require that the fields on the brane couple to $\gamma_{\mu \nu}$ rather than to $h_{\mu \nu}$. Now, let the Hubble parameter and the energy momentum tensor, with respect to $\gamma_{\mu \nu}$, be $\mathcal{H}=\partial_{\xi} \ln E$ and $S_{\mu \nu}$ respectively. These are related to $H$ and $T_{\mu \nu}$ through the relations

$$
H=-(D-2) e^{-2 \phi /(D-2)} \mathcal{H}, \quad T_{\mu \nu}=e^{4 \phi /(D-2)} S_{\mu \nu} .
$$

It then follows that Eq. (4.2) can be written in the standard form

$$
\frac{d \mu}{d \xi}+(D-2)(\mu+p) \mathcal{H}=0
$$

We now look at Eqs. (4.1)-(4.3) for some relevant values of $\omega=p / \mu$.

\subsection{Pure tension: $\omega=-1$}

In this case $T^{\phi}=0$ and $\mu=\mu_{0}=$ const, hence $H^{2}=\frac{\mu_{0}^{2}}{4}-\lambda^{2}$, which in general corresponds to an accelerating de Sitter phase. In our case, however, we have $\mu_{0}^{2}=$ $4 \lambda^{2}$. Indeed, given the definition (B.3), we see that the condition $T^{\phi}=0$ implies that $\mathcal{L}=-\Lambda e^{2 \phi(z)}$, for some constant $\Lambda$. In turn, this yields $\mu_{0}=\Lambda e^{2 \phi(z(\tau))}$. Hence, the vacuum energy density is constant only if the brane is static, i.e. $\dot{z}=H=0$. The equality $\mu_{0}^{2}=4 \lambda^{2}$ implies that

$$
\mu_{0}^{2}=\frac{16 V_{0}}{D-1} e^{\beta \bar{\phi}_{0}}
$$

and this shows that the brane vacuum energy density is arbitrary. In fact, for any value of the parameter $\beta$, the shifted dilaton "vacuum" $\bar{\phi}_{0}$ can always be adjusted so that (4.8) is satisfied, without affecting the Poincaré invariance of the solution. Thus, we could consider this as a self tuning mechanism, pretty much along the same lines of [3]. The main differences with respect to [3] is that here we work in string frame and, by including a coupling to the shifted dilaton, we find a completely smooth, anti-de Sitter bulk. The relation (4.8) appears to leave an ambiguity about the sign of the energy density. However, by using the $i j$ component of Eq. (3.1) with $T^{\phi}=0$, we find that $2 \sigma^{\prime}=p$. Hence, for $\sigma=\lambda\left(z-z_{0}\right), p$ is positive and the tension

\footnotetext{
${ }^{3}$ Note, that our evolution equation above can also be seen as a special case of the generic set of energy-non-conserving brane cosmologies studied in [23].
} 
is negative. On the other hand, $p$ is negative and $\mu$ is positive in the dual case, in agreement with the transformation laws (4.4).

The dual solutions differ not only in the sign of the tension, but also in the effective Planck mass. By assuming for simplicity that the brane is located at $z=0$, the latter is given by

$$
M_{P L}^{D-3} \sim \int_{-\infty}^{0} d z e^{(D-3) \sigma} e^{-2 \phi}=\int_{-\infty}^{0} d z e^{-2 \sigma}
$$

For the solution $\sigma=\lambda z$, the perturbative expansion (governed by $e^{\phi}$ ) is under control but the Planck mass diverges, and gravity is not localised on the brane. The dual solution $\tilde{\sigma}=-\lambda z$ yields instead a finite Planck mass, but the string coupling now is strong. We will comment about these results in the last section. For the moment, we observe that also in Jordan frame the solution is static. Indeed, the Friedmann equation, for pure tension, becomes

$$
\mathcal{H}^{2}=\frac{\mu_{0}^{2}-4 \lambda^{2}}{4 a(D-2)^{2}} e^{4 \phi\left(z_{b}(\tau)\right) /(D-2)}
$$

where $z_{b}(\tau)$ denotes the time-dependent location of the brane. This equation yields again non-static solutions whenever $\mu_{0}^{2}>4 \lambda^{2}$. However, given that, changing to Jordan frame, $\mu$ and $p$ are the same, we still have the condition $\dot{z}_{b}=0$, which in turn implies that $\mu_{0}^{2}=4 \lambda^{2}$.

In conclusion, when $\omega=-1$, only static solutions are allowed. The vacuum energy on the brane is determined in terms of the bulk AdS radius through $\lambda$, and it is invariant under T-duality. Since $\lambda \neq 0$, the brane vacuum energy cannot vanish, but the solution is always Poincaré invariant.

\subsection{Perfect fluid: $\omega \neq-1$}

In this case, we have $T^{\phi}=-2 \mu(1+\omega)$ and the evolution equation yields

$$
\mu=\mu_{0} e^{(1+\omega) \sigma}
$$

This can be plugged into the Friedmann equation (4.1), leading to

$$
\dot{z}_{b}^{2}(\tau)=\frac{\mu_{0}^{2}}{4 \lambda^{2}} e^{2(1+\omega) \lambda\left(z_{b}(\tau)-z_{0}\right)}-1
$$

When the brane moves towards increasing values of $z$, and for $\sigma=\lambda\left(z_{b}(\tau)-z_{0}\right)$, we find the solution

$$
z_{b}(\tau)-z_{0}=\frac{1}{2 \lambda(1+\omega)} \ln \left\{\frac{4 \lambda^{2}}{\mu_{0}^{2}}\left[1+\tan ^{2}\left((1+\omega) \lambda\left(\tau-\tau_{0}\right)\right)\right]\right\}
$$


for arbitrary $\tau_{0}$. It follows that the scale factor and the energy density are given respectively by

$$
\begin{aligned}
& A(\tau)=\left(\frac{2 \lambda}{\left|\mu_{0}\right|}\right)^{\frac{1}{1+\omega}}\left[1+\tan ^{2}\left((1+\omega) \lambda\left(\tau-\tau_{0}\right)\right)\right]^{\frac{1}{2(1+\omega)}} \\
& \mu(\tau)=-2 \lambda\left[1+\tan ^{2}\left((1+\omega) \lambda\left(\tau-\tau_{0}\right)\right)\right]^{\frac{1}{2}}
\end{aligned}
$$

We note that when $\omega>-1$, the scale factor has an absolute minimum at $\tau=\tau_{0}$, which corresponds to $\mu\left(\tau_{0}\right)=-2 \lambda$. If we were to make the further assumption $\mu\left(\tau_{0}\right) \equiv \mu_{0}$, then $A\left(\tau_{0}\right)=1$ and the bounce would be located at $z_{0}$. It is easy to see that this solution corresponds to a bouncing brane cosmology ${ }^{4}$. Indeed the scale factor $A(\tau)$ smoothly goes from a contracting phase to an expanding one, passing through a positive minimum value at $\tau=\tau_{0}$. Also, $\dot{H}>0$ for all $\tau$, hence the Universe is superinflating at all times. The string coupling is under control, but the brane has negative tension.

The dual solution can be easily obtained with the substitutions $\lambda \rightarrow-\lambda, \omega \rightarrow \tilde{\omega}$, and $\mu_{0} \rightarrow-\tilde{\mu}_{0}=-2 \lambda$ in the equations above. The form of the Friedmann equation does not change, and its solution is

$$
z_{b}(\tau)-\tilde{z}_{0}=-\frac{1}{2 \lambda(1+\tilde{\omega})} \ln \left[1+\tan ^{2}\left((1+\tilde{\omega}) \lambda\left(\tau-\tau_{0}\right)\right)\right] .
$$

However, the dual scale factor is defined by

$$
\tilde{A}(\tau) \equiv A(\tau)^{-1}=e^{-\lambda\left(z_{b}(\tau)-z_{0}\right)}
$$

which leads to

$$
\tilde{A}(\tau)=\left[1+\tan ^{2}\left((1+\tilde{\omega}) \lambda\left(\tau-\tau_{0}\right)\right)\right]^{\frac{1}{2(1+\tilde{\omega})}}
$$

while the energy density reads

$$
\tilde{\mu}(\tau)=2 \lambda\left[1+\tan ^{2}\left((1+\tilde{\omega}) \lambda\left(\tau-\tau_{0}\right)\right)\right]^{\frac{1}{2}} .
$$

Again, the dual solution still describes a bouncing Universe, and, provided $\tilde{\omega}>-1$, the dual scale factor reaches the minimum $\tilde{A}\left(\tau_{0}\right)=1$ at the bounce location $\tilde{z}_{0}$. However, the energy density is now positive, but the string coupling is strong.

As mentioned above, the duality transformations (4.4) imply a violation of the strong energy condition. As in the static case, the solution associated to $\sigma\left(z_{b}\right)$ corresponds to a negative energy density on the brane. To cope with this problem, we follow the model proposed in [8], and we assume that

$$
\mu=\rho+F, \quad p=\pi-F,
$$

\footnotetext{
${ }^{4}$ See [24 for a recent detailed study on string inspired bouncing cosmologies.
} 
where $\rho, \pi$, and $F$ are treated as functions of $\tau$. By further imposing that $\tilde{\rho}=\rho$ and $\tilde{\pi}=\pi$, we separate a T-dual invariant matter contribution from a time-dependent tension. From Eqs. (4.4) it then follows that $\tilde{F}=-F-2 \rho$. If also the matter on the brane behaves as a perfect fluid, i.e. $\pi=\gamma \rho$, then we have

$$
\rho(\tau)=\frac{1+\omega}{1+\gamma} \mu(\tau)
$$

where $\mu(\tau)$ is given by Eq. (4.15). Thus, provided $\omega<-1$, and $\gamma>-1$, we ensure that $\rho$ is always positive. When $\pi=\rho=0$, the only source of stress is the tension $F(\phi)$, hence, under T-duality, we simply have $\tilde{F}=-F$, as in the static case. This shows that even in the simplest case, the insertion of a brane in the background breaks the physical equivalence of dual bulks mentioned in Sec. 2 .

\subsection{Pre-Big Bang Scenario}

We now come to the implementation of the PBB scenario in our model. For simplicity, we consider the case when $-\mu_{0}=\tilde{\mu}_{0}=2 \lambda$, and we assume that $\tilde{\omega}>-1$. Using the duality identity $-(\omega+1)=\tilde{\omega}+1$, we compare Eqs. (4.13) and (4.16), and we find that $z_{0}=\tilde{z}_{0}$. Then, we construct a global solution to the Friedmann equation such that

$$
\begin{array}{ll}
z_{b}(\tau)=z_{0}+\frac{1}{2 \lambda(\omega+1)} \ln \left[1+\tan ^{2}\left((1+\omega) \lambda\left(\tau-\tau_{0}\right)\right)\right], & \tau<\tau_{0}, \\
z_{b}(\tau)=z_{0}-\frac{1}{2 \lambda(\tilde{\omega}+1)} \ln \left[1+\tan ^{2}\left((1+\tilde{\omega}) \lambda\left(\tau-\tau_{0}\right)\right)\right], & \tau>\tau_{0},
\end{array}
$$

i.e. we patch at the bounce the solutions (4.13) and (4.16), respectively. The global scale factor, for a fixed value of $\tilde{\omega}$, becomes

$$
A(\tau)= \begin{cases}{\left[1+\tan ^{2}\left((1+\tilde{\omega}) \lambda\left(\tau-\tau_{0}\right)\right)\right]^{\frac{-1}{2(1+\tilde{\omega})}},} & \tau<\tau_{0}, \\ {\left[1+\tan ^{2}\left((1+\tilde{\omega}) \lambda\left(\tau-\tau_{0}\right)\right)\right]^{\frac{1}{2(1+\tilde{\omega})}},} & \tau>\tau_{0},\end{cases}
$$

and we see that it is always growing, with a smooth inflection point at $\tau=\tau_{0}$. Analogously, the global Hubble parameter reads

$$
H(\tau)=\left\{\begin{aligned}
-2 \lambda \tan \left((1+\tilde{\omega}) \lambda\left(\tau-\tau_{0}\right)\right), & \tau<\tau_{0}, \\
2 \lambda \tan \left((1+\tilde{\omega}) \lambda\left(\tau-\tau_{0}\right)\right), & \tau>\tau_{0},
\end{aligned}\right.
$$

and thus $\dot{H}$ turns from negative to positive at the bounce, revealing a superinflationary behaviour for $\tau>\tau_{0}$. However, exploiting the results of the static case discussed above, it is clear that brane gravity is not localised in the pre-bounce phase whereas 
it is in the post-bounce one. Moreover, while in the pre-bounce phase the string coupling is weak, in the post-bounce it becomes strong, and the model might become unreliable because of string loop corrections. However, note that if we interpret $\bar{\phi}$ as the reduced string coupling constant [21], the latter might be under control as $\bar{\phi}=\bar{\phi}_{0}$.

In fact, in order to achieve localisation of gravity before the bounce, one would have to modify the Eq. (4.20), as

$$
\mu(\tau)=-\rho(\tau)+F(\tau), \quad p(\tau)=-\pi(\tau)-F(\tau)
$$

so that $\tilde{\omega}<-1$. Then, the global scale factor and Hubble parameter can be obtained by the expressions above by simply replacing $\tilde{\omega}$ with $\omega$.

In both cases, this construction violates the positivity of the total energy density on the brane. Note that this violation is not localised around $z_{0}$, but it holds for all times before (or after) the dual transition, in opposition to the PBB scenario, where the violation (if any) is always localised.

\section{Discussion}

We have considered a setup where a codimension-one brane is embedded in a static bulk, and we assumed T-duality symmetry along the time direction, as well as the space directions parallel to the brane. The time-like T-duality is shown to be consistent as the bulk geometry is anti-de Sitter.

When the brane is static, there are smooth solutions, which show Poincaré invariance on the brane, regardless of the value of its tension. This is possible thanks to a self-tuning property of the bulk shifted-dilaton vacuum energy. We also found brane-moving solutions, which lead to both bouncing and PBB-like cosmology. In the latter case, we found that gravity can be localised on the brane via T-duality, however the model incurs a violation of the strong energy condition.

In all these cases, regular solutions for which the string coupling is under control do not lead to localisation of gravity on the brane. This is not a priori bad news. Indeed, gravity could be induced on the brane via quantum effects, as discovered in [25]. Note, in fact, that including an Einstein-Hilbert term on the brane does not require (at least in the static case) a modification of the $\mathrm{AdS}_{5}$ background studied here, the latter being 4D flat. Moreover, the van Dam-Veltman-Zakharov discontinuity of the graviton propagator, present in the 5D flat case [25], might be absent here, where the background is AdS [26]. It also appears that previously found no-go theorems [7, 10] for self-tuning RS backgrounds, do not apply here as the

background we found is completely smooth. However, similar setups studied in the past, in Einstein frame, seem to require conformality of the localised matter on the brane [27], and it is not a priori obvious whether such consistency conditions are 
present in our setup as well. Another way of inducing $4 \mathrm{D}$ gravity on the brane is via bulk higher-curvature terms [28]; however, this might spoil the self-tuning feature in such a codimension-one model [6, 29].

Besides these issues, the model studied in this Letter can be extended in other ways. For instance, one can consider the embedding of a second brane, which might regularise the effective Planck mass. Also, a second brane could cure the singularities, mentioned in Sec. (2), that appear in the bulk when the shifted dilaton is not constant. Finally, one might consider including in the bulk the NS anti-symmetric tensor field, along the lines of some PBB models.

Generally speaking, time-like T-duality is a symmetry not yet fully explored in the context of string cosmology, and it might be worth investigating. As an example, the inclusion of time-like duality in the String Gas Cosmology of Brandenberger and Vafa [30], might lead to interesting insights.

\section{Acknowledgments}

The work of OC has been partly supported by the EC commission via the FP5 grant HPRN-CT-2002-00325. OC is grateful to the University of Padova for financial support via the PRIN-2003 project. We wish to thank D. Wands and M. Gasperini for useful suggestions, and P. Watts for reading the manuscript.

\section{A. Extrinsic curvature and its dual}

In this appendix we study how the extrinsic curvature transforms under T-duality. We consider a brane with (D-1)-dimensional induced metric

$$
h_{\mu \nu} d x^{\mu} d x^{\nu}=-d \tau^{2}+e^{2 \sigma(z)} \delta_{i j} d x^{i} d x^{j}
$$

embedded in a D-dimensional bulk space-time with metric

$$
g_{A B} d x^{A} d x^{B}=e^{2 \sigma(z)} \eta_{\mu \nu} d x^{\mu} d x^{\nu}+d z^{2}
$$

where $\eta_{\mu \nu}$ denotes a (D-1)-dimensional Minkowski metric. We follow the conventions of [31], and we assume that the brane moves in the bulk with velocity $v^{A}=$ $\left(\dot{t}, 0,0, \ldots, 0, \dot{z}_{b}\right)$, where $z_{b}(\tau)$ is the brane location in the bulk, and the dot stands for differentiation with respect to $\tau$. Our choice for the induced metric implies that $\dot{t}= \pm e^{-\sigma} \sqrt{1+\dot{z}_{b}^{2}}$. The vector $n^{A}$ normal to the brane is defined such that

$$
g_{A B} n^{A} n^{B}=1, \quad n_{A} v^{A}=0 .
$$

These conditions yield the components of $n^{A}$, namely

$$
n_{A}=\left( \pm e^{\sigma} \dot{z}_{b}, 0,0, \ldots, 0, \mp \sqrt{1+\dot{z}_{b}^{2}}\right) .
$$


If we choose $(+,-)$, we ensure that the normal vector points into the bulk ${ }^{5}$. The extrinsic curvature is defined as

$$
K_{\mu \nu}=\frac{\partial X^{A}}{\partial x^{\mu}} \frac{\partial X^{B}}{\partial x^{\nu}} \nabla_{A} n_{B}
$$

where $X^{A}\left(x^{\mu}\right)$ are the bulk (brane) coordinates. In our case, its components are given by

$$
K_{i j}=-\sigma^{\prime} \sqrt{1+\dot{z}_{b}^{2}} h_{i j}, \quad K_{\tau \tau}=e^{-\sigma} \frac{d}{d z_{b}} e^{\sigma} \sqrt{1+\dot{z}_{b}^{2}}
$$

hence the trace reads

$$
K_{\mu \nu} h^{\mu \nu}=K=-(D-1) \sigma^{\prime} \sqrt{1+\dot{z}_{b}^{2}}-\frac{d}{d z_{b}} \sqrt{1+\dot{z}_{b}^{2}}
$$

The T-dual bulk and induced metrics considered in this work are obtained by replacing $\sigma$ with $-\sigma$. The velocity vector can still be defined as $v^{A}=\left(\dot{t}, 0,0, \ldots, 0, \dot{z}_{b}\right)$, while the normalisation condition on $\dot{t}$ now becomes $\dot{t}= \pm e^{\sigma} \sqrt{1+\dot{z}_{b}^{2}}$. By repeating the same computations as above (with the same choice for the signs) it is easy to show that the dual normal vector becomes

$$
\tilde{n}_{A}=\left(e^{-\sigma} \dot{z}_{b}, 0,0, \ldots, 0,-\sqrt{1+\dot{z}_{b}^{2}}\right)
$$

while the components of the extrinsic curvature read

$$
\tilde{K}_{i j}=\sigma^{\prime} \sqrt{1+\dot{z}_{b}^{2}} \tilde{h}_{i j}, \quad \tilde{K}_{\tau \tau}=e^{\sigma} \frac{d}{d z_{b}} e^{-\sigma} \sqrt{1+\dot{z}_{b}^{2}} .
$$

Thus, the relevant T-duality transformations read

$$
\begin{aligned}
& n_{t} \stackrel{\mathrm{T}}{\longrightarrow} \tilde{n}_{t}=e^{-2 \sigma} n_{t}, \quad n_{z} \stackrel{\mathrm{T}}{\longrightarrow} \tilde{n}_{z}=n_{z}, \\
& K_{i j} \stackrel{\mathrm{T}}{\longrightarrow} \tilde{K}_{i j}=-e^{-4 \sigma} K_{i j} \\
& \tilde{K}_{\tau \tau} \stackrel{\mathrm{T}}{\longrightarrow} \tilde{K}_{\tau \tau}=K_{\tau \tau}-2 \sigma^{\prime} \sqrt{1+\dot{z}_{b}^{2}}, \\
& K \stackrel{\mathrm{T}}{\longrightarrow} \tilde{K}=K+2(D-1) \sigma^{\prime} \sqrt{1+\dot{z}_{b}^{2}} .
\end{aligned}
$$

\footnotetext{
${ }^{5}$ Here we also choose $\dot{t}>0$, and this leads to the condition $\operatorname{sign}\left(n^{t}\right)=\operatorname{sign}\left(n^{z}\right)$. If instead one takes $\dot{t}<0$, then $\operatorname{sign}\left(n^{t}\right)=-\operatorname{sign}\left(n^{z}\right)$.
} 


\section{References}

[1] L. Randall and R. Sundrum, Phys. Rev. Lett. 83, 3370 (1999) [arXiv:hep-ph/9905221].

[2] L. Randall and R. Sundrum, Phys. Rev. Lett. 83, 4690 (1999) [arXiv:hep-th/9906064].

[3] S. Kachru, M. B. Schulz and E. Silverstein, Phys. Rev. D 62, 045021 (2000) [arXiv:hep-th/0001206].

[4] H. A. Chamblin and H. S. Reall, Nucl. Phys. B 562 (1999) 133 [arXiv:hep-th/9903225].

[5] A. Krause, JHEP 0309, 016 (2003) [arXiv:hep-th/0007233];

A. Kehagias and K. Tamvakis, Mod. Phys. Lett. A 17 (2002) 1767 [arXiv:hep-th/0011006];

J. E. Kim, B. Kyae and H. M. Lee, Phys. Rev. Lett. 86 (2001) 4223 [arXiv:hep-th/0011118].

[6] I. Low and A. Zee, Nucl. Phys. B 585 (2000) 395 [arXiv:hep-th/0004124].

[7] C. Csaki, J. Erlich, C. Grojean and T. J. Hollowood, Nucl. Phys. B 584 (2000) 359 [arXiv:hep-th/0004133].

[8] G. T. Horowitz, I. Low and A. Zee, Phys. Rev. D 62 (2000) 086005 [arXiv:hep-th/0004206].

[9] D. Langlois and M. Rodriguez-Martinez, Phys. Rev. D 64 (2001) 123507 [arXiv:hep-th/0106245].

[10] J. M. Cline and H. Firouzjahi, Phys. Rev. D 65 (2002) 043501 [arXiv:hep-th/0107198].

[11] M. Rinaldi, Phys. Lett. B 582, 249 (2004) [arXiv:hep-th/0311147].

[12] M. Rinaldi and P. Watts, JCAP 0503 (2005) 006 [arXiv:hep-th/0411185].

[13] T. H. Buscher, Phys. Lett. B 194 (1987) 59.

[14] M. Gasperini and G. Veneziano, Phys. Rept. 373, 1 (2003) [arXiv:hep-th/0207130].

[15] J. E. Lidsey, D. Wands and E. J. Copeland, Phys. Rept. 337, 343 (2000) [arXiv:hep-th/9909061].

[16] C. M. Hull, JHEP 9807 (1998) 021 [arXiv:hep-th/9806146].

[17] D. L. Welch, Phys. Rev. D 50 (1994) 6404 [arXiv:hep-th/9405070].

[18] R. Argurio, arXiv:hep-th/9807171. 
[19] K. A. Meissner and G. Veneziano, Mod. Phys. Lett. A 6 (1991) 3397 [arXiv:hep-th/9110004].

K. A. Meissner and G. Veneziano, Phys. Lett. B 267 (1991) 33.

[20] M. Gasperini and G. Veneziano, Astropart. Phys. 1 (1993) 317 [arXiv:hep-th/9211021].

M. Gasperini, J. Maharana and G. Veneziano, Nucl. Phys. B 472 (1996) 349 [arXiv:hep-th/9602087].

[21] M. Gasperini, M. Giovannini and G. Veneziano, Phys. Lett. B 569 (2003) 113 [arXiv:hep-th/0306113].

M. Gasperini, M. Giovannini and G. Veneziano, Nucl. Phys. B 694 (2004) 206 [arXiv:hep-th/0401112].

[22] C. Barcelo and M. Visser, Phys. Rev. D 63, 024004 (2001) [arXiv:gr-qc/0008008];

S. Foffa, Phys. Rev. D 66, 063512 (2002) [arXiv:hep-th/0207103].

[23] E. Kiritsis, G. Kofinas, N. Tetradis, T. N. Tomaras and V. Zarikas, JHEP 0302 (2003) 035 [arXiv:hep-th/0207060].

[24] T. Biswas, A. Mazumdar and W. Siegel, arXiv:hep-th/0508194.

[25] G. R. Dvali, G. Gabadadze and M. Porrati, Phys. Lett. B 485 (2000) 208 [arXiv:hep-th/0005016]; see G. Gabadadze and A. Iglesias, arXiv:hep-th/0407049, for a more recent nonperturbative investigation on such models.

[26] M. Porrati, Phys. Lett. B 498 (2001) 92 [arXiv:hep-th/0011152].

[27] Z. Kakushadze, Mod. Phys. Lett. A 15 (2000) 1879 [arXiv:hep-th/0009199].

[28] O. Corradini, A. Iglesias, Z. Kakushadze and P. Langfelder, Phys. Lett. B 521 (2001) 96 [arXiv:hep-th/0108055];

O. Corradini, Mod. Phys. Lett. A 20 (2005) 2775 [arXiv:hep-th/0405038].

[29] P. Binetruy, C. Charmousis, S. C. Davis and J. F. Dufaux, Phys. Lett. B 544 (2002) 183 [arXiv:hep-th/0206089].

[30] R. H. Brandenberger and C. Vafa, Nucl. Phys. B 316 (1989) 391;

S. Alexander, R. H. Brandenberger and D. Easson, Phys. Rev. D 62 (2000) 103509 [arXiv:hep-th/0005212].

[31] P. Brax, D. Langlois and M. Rodriguez-Martinez, Phys. Rev. D 67, 104022 (2003) [arXiv:hep-th/0212067]. 